\title{
Blunt prenatal trauma resulting in fetal epidural or subdural hematoma: case report and systematic review of the literature
}

\author{
Jacob R. Joseph, MD, Brandon W. Smith, MD, and Hugh J. L. Garton, MD \\ Department of Neurosurgery, University of Michigan, Ann Arbor, Michigan
}

Blunt prenatal trauma is known to have consequences to the developing brain, and can result in subdural hematoma $(\mathrm{SDH})$ or epidural hematoma $(\mathrm{EDH})$. The authors present a case of blunt prenatal trauma resulting in a fetal SDH, intraparenchymal hematoma, and intraventricular hemorrhage, and perform a systematic review of the literature. This systematic review was conducted according to the PRISMA (Preferred Reporting Items for Systematic Reviews and Meta-Analyses) guidelines. Relevant studies (up to April 2016) that reported on cases of fetal SDH or EDH after blunt prenatal trauma were identified from the PubMed database. The primary outcome was fetal mortality, and the secondary outcome was neurological outcome. Fourteen studies were included in the analysis, comprising a total of 14 patients including the present case. The average gestational age at discovery of hemorrhage was 30.1 weeks. Nine mothers were in a motor vehicle collision and 3 were assaulted; the mechanism of injury for 2 mothers was not defined. Twelve patients had SDH, 1 had EDH, and 1 had conflicting reports. Three patients had intrauterine fetal demise, and 3 died in the neonatal period after birth. Three patients had persistent neurological deficit, and 5 were neurologically intact. Fetal SDH or $\mathrm{EDH}$ after blunt trauma to the mother trauma is rare and is associated with mortality. However, a significant number of patients can have good neurological outcomes.

http://thejns.org/doi/abs/10.3171/2016.7.PEDS16282

KEY WORDS blunt trauma; epidural hematoma; fetal hematoma; prenatal trauma; subdural hematoma

$\mathrm{T}$ RaUMA is the leading nonobstetric cause of maternal death among pregnant women, and complicates as many as 1 in 12 pregnancies. ${ }^{18,20}$ Damage to the developing brain is a known consequence of maternal trauma, regardless of severity. ${ }^{6}$ Most potential damage to the brain is indirect, as a result of stroke, periventricular leukomalacia, or intraventricular hemorrhage (IVH). ${ }^{2,14} \mathrm{How}-$ ever, the risk of direct damage resulting in skull fractures, epidural hematoma $(\mathrm{EDH})$, or subdural hematoma (SDH) is also present. We present a case of trauma occurring in a mother that resulted in fetal SDH, intraparenchymal hematoma, and IVH. We also perform a systematic review to evaluate all reported patients who had blunt prenatal trauma resulting in fetal $\mathrm{EDH}$ or $\mathrm{SDH}$.

\section{Case Report}

A 28 -year-old pregnant woman in her 35th week of gestation was involved in a motor vehicle collision (MVC) during which the driver's side was "T-boned" at approxi- mately 45 miles per hour. She was immediately transported to a trauma center where she was noted to have a "seat belt sign," with abdominal bruising noted along her abdomen. She was also found to have several soft-tissue contusions and nonoperative orthopedic injuries. She complained of abdominal tenderness and contractions. Due to both maternal and fetal tachycardia, indicative of fetal distress, an emergent cesarean section was performed. The patient's Apgar scores were 4, 6, and 8 at 1,5, and 10 minutes of life, respectively. At 12 hours of life, the infant was noted to have several episodes of posturing, consisting of extension and internal rotation of her arms and legs, with bradycardia and oxygen desaturation. On Day 1 of life, she had a 10-minute seizure with eye deviation and clonic jerking of bilateral lower extremities. The seizure was successfully treated with lorazepam and phenobarbital.

A cranial ultrasound image showed a bithalamic echogenicity that was more prominent on the left side and measured $1.7 \mathrm{~cm}$ in greatest dimension (Fig. 1). Subsequent 


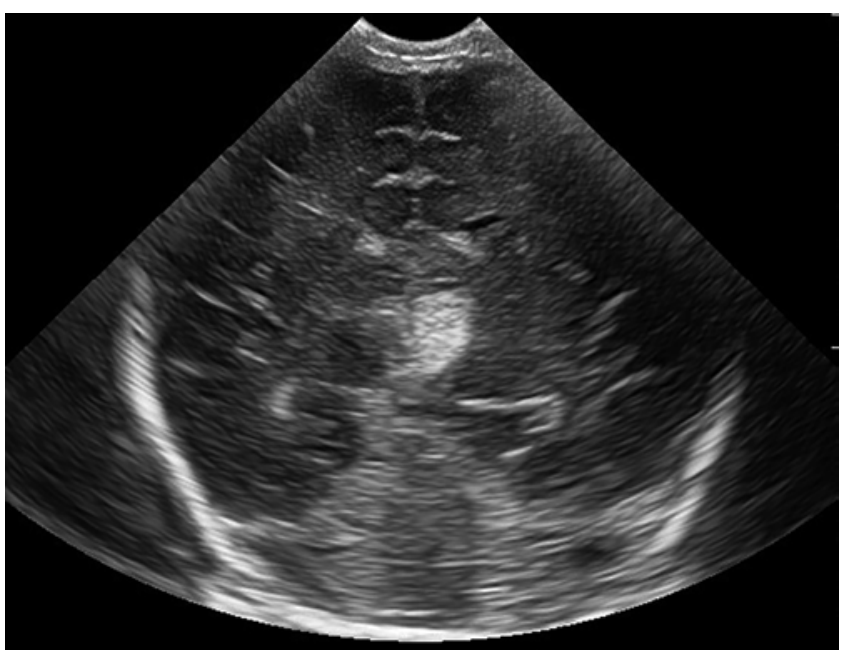

FIG. 1. Coronal cranial ultrasound showing bithalamic echogenicity consistent with hemorrhage.

MRI demonstrated bilateral SDHs, subarachnoid and subpial hemorrhages consistent with contusions in the bilateral temporal lobes and the left parietooccipital region, and IVH. The previously seen bithalamic echogenicities were also confirmed to be intraparenchymal hemorrhages (Fig. 2). Mild ventriculomegaly was also noted.

Electroencephalography was performed for 3 days following the initial event and did not reveal any further seizure activity. Results of the physical examination were un-

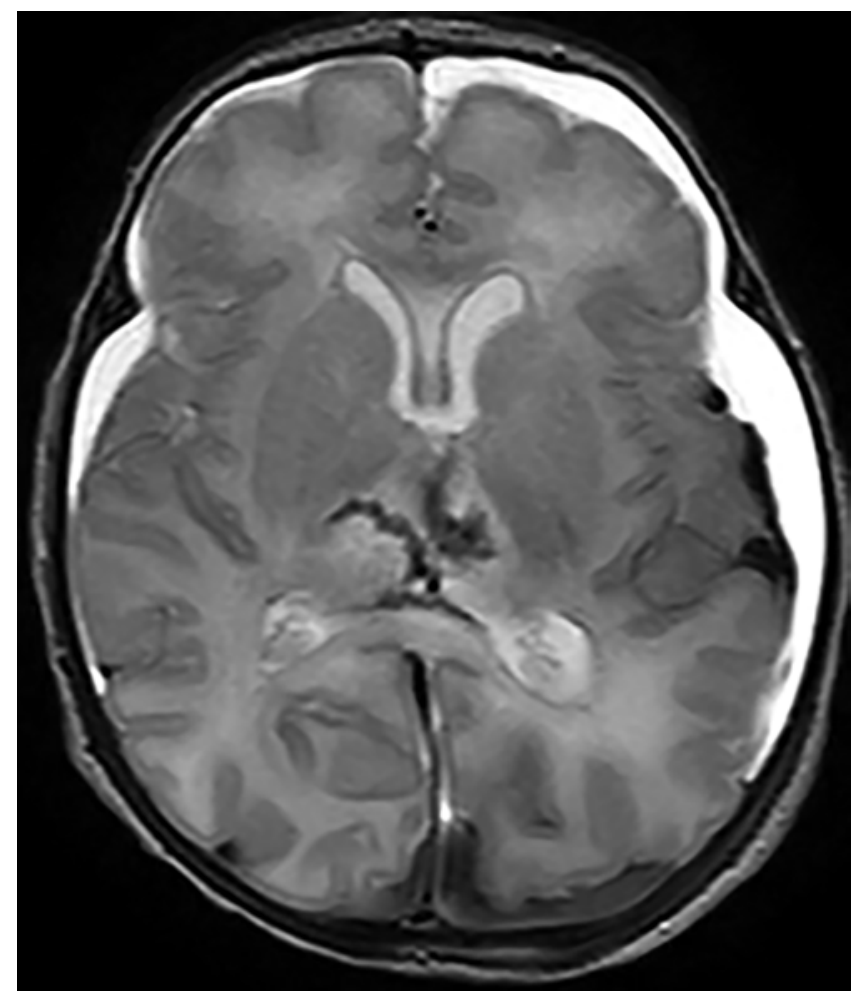

FIG. 2. Axial T2-weighted MR image demonstrating bithalamic hemorrhage, bilateral SDH, and left parietooccipital subarachnoid/subpial hemorrhage. remarkable, with full strength noted in all extremities and a soft, sunken fontanelle. The patient was discharged to home on Day 8 of life in stable condition. She was maintained on phenobarbital, with plans to be weaned off the medication at 3-4 months. Repeat MRI performed at 2 weeks of age showed an interval decrease in the subdural, intraparenchymal, and subpial hemorrhages (Fig. 3). At last follow-up (3 months of age), the patient demonstrated no known neurological deficits or developmental delay.

\section{Methods}

\section{Literature Search and Inclusion Criteria}

This systematic review was conducted according to PRISMA (Preferred Reporting Items for Systematic Reviews and Meta-Analyses) guidelines. ${ }^{19}$

We identified relevant articles published up to April 2016 through a search of the PubMed database. The initial search strategy for publications was performed using the search terms fetal AND intracranial AND trauma. Subsequently, several search strategies were performed to identify any further relevant studies. These included fetal AND (subdural OR extradural OR epidural) AND hematoma (epidural OR subdural OR subarachnoid OR intraventricular OR intracranial) AND hemorrhage AND (intrauterine OR fetus) AND trauma. In addition, manual checks of the reference lists were performed. Only articles written in the English language were included. After the initial search, all titles and abstracts were reviewed. Finally, each article that met criteria underwent full-text review. Articles that did not have full text were excluded. Reports

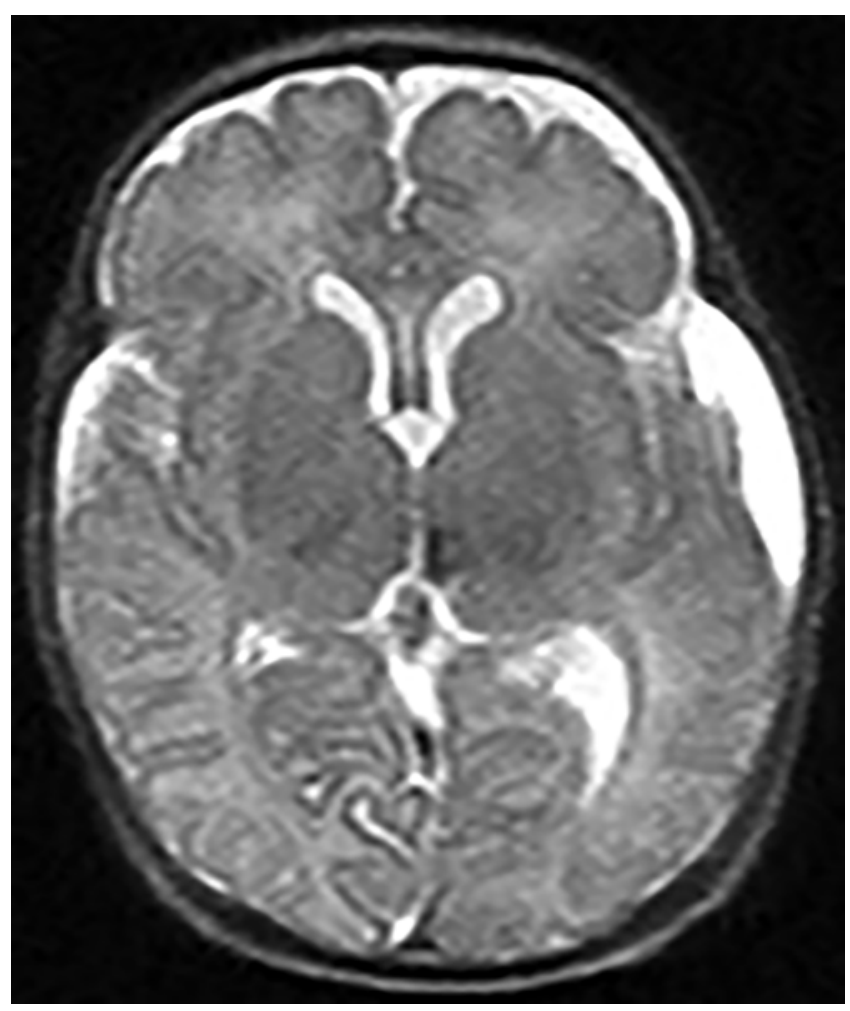

FIG. 3. Axial T2-weighted MR image demonstrating interval decrease in bithalamic hemorrhage and in size of SDH. 


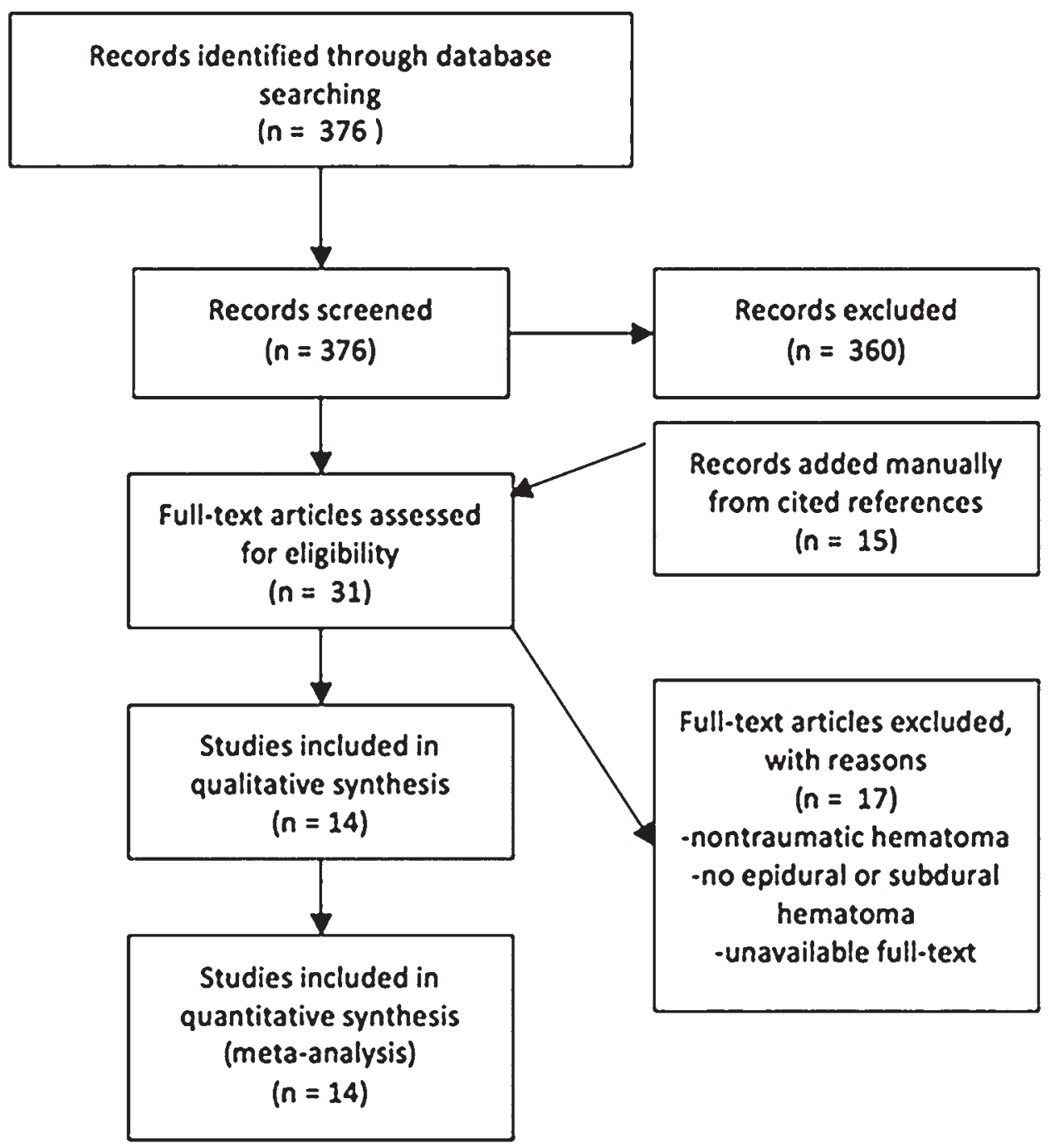

FIG. 4. Flow diagram showing selection of the studies for this systematic review of blunt prenatal trauma resulting in fetal SDH or $\mathrm{EDH}$.

that did not involve trauma or extraaxial hemorrhages were excluded. Penetrating trauma was also excluded. Descriptions of combined extraaxial and intraparenchymal hemorrhages were not excluded.

\section{Data Extraction and Outcome Measures}

We extracted the following data from each report: first author name, last author name, date of publication, gestational age at discovery of intracranial hemorrhage, mechanism of trauma, seat belt use, air bag deployment, other traumatic injuries to the fetus, traumatic injuries to the mother, maternal surgery, method of birth, hemorrhage type, intervention for intracranial hemorrhage, and outcome. Risk of bias was evaluated for each study at the time of data extraction, and it was noted that there was a risk for publication bias in the present study. Mortality was the primary outcome of the study, and secondary outcome was neurological disability. It was noted that followup time was variable between studies, as were details in reports of neurological outcome.

\section{Results}

\section{Study Selection}

A total of 376 studies were identified through database searches and underwent review of titles and abstracts. An additional 15 studies were found manually through review of reference lists. Three hundred sixty studies were excluded after title and abstract review. Reasons for exclusion included irrelevance to the present study. Thirty-one studies were deemed to be eligible for full-text review. After full-text review, 17 studies were excluded. Reasons for exclusion included lack of EDH or SDH, nontraumatic hemorrhage, and full-text availability. A total of 14 studies were included in the final analysis., 3,7-10,13,15-17,21,22,24,26 Of note, 2 studies described the same case and were combined for the purposes of this study. ${ }^{17,21}$ The search flow diagram is shown in Fig. 4.

\section{Study Characteristics and Outcomes}

A total of 14 patients were identified, including the 

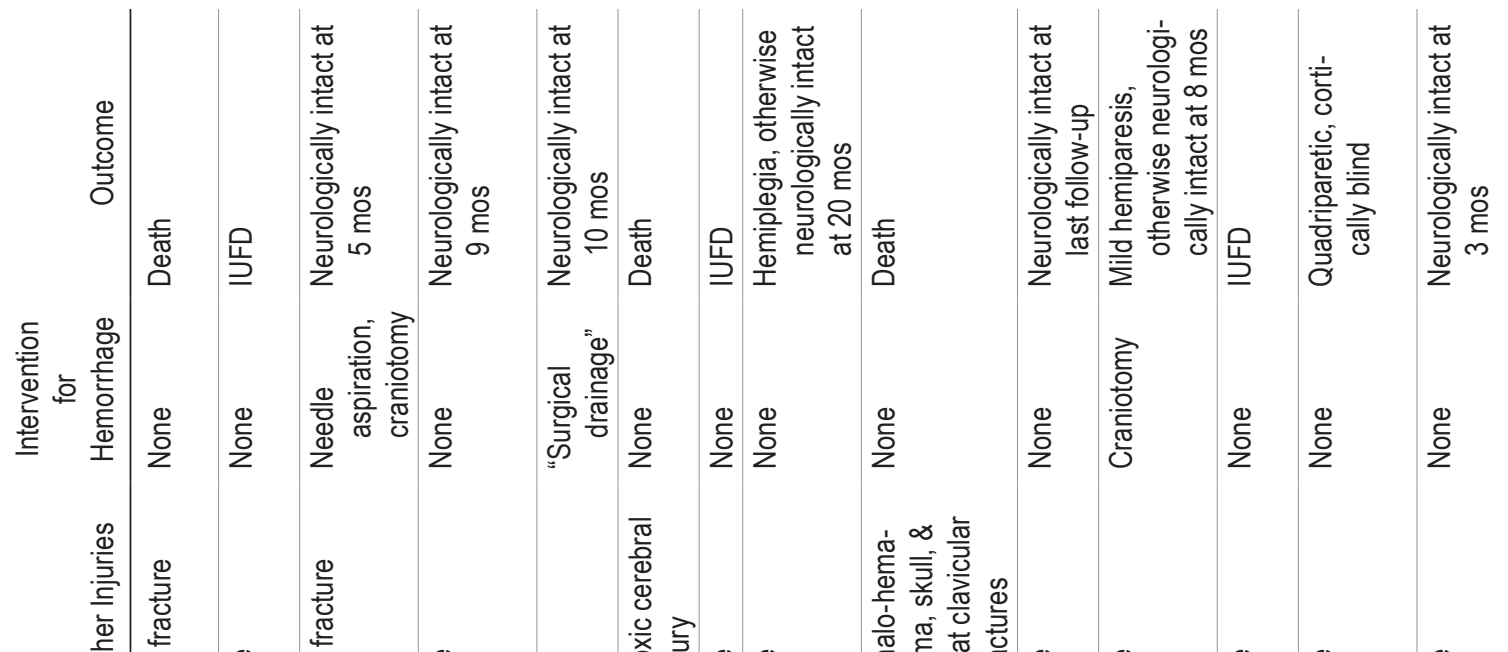

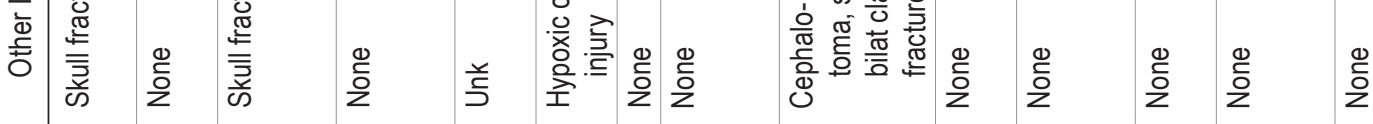

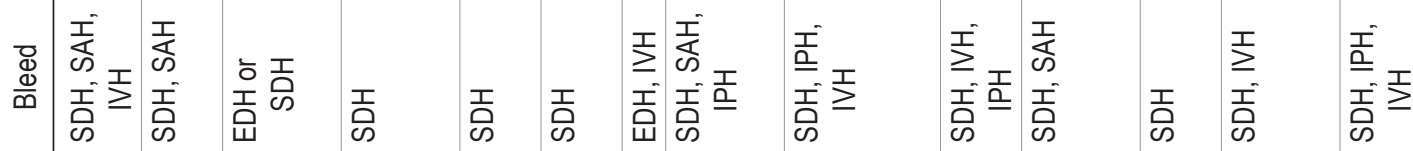

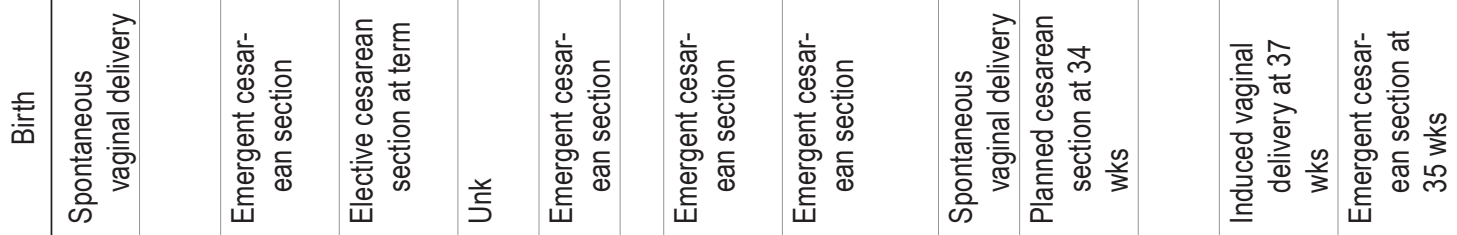

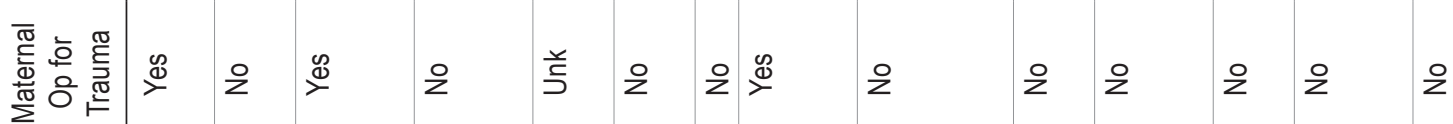

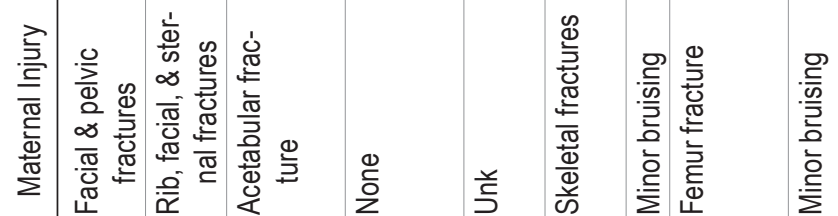

$\stackrel{\infty}{\stackrel{0}{2}} \stackrel{0}{\overline{5}}$

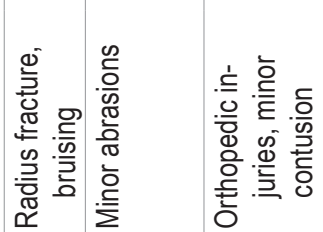

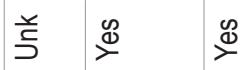

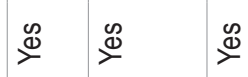

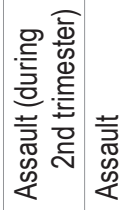

న প

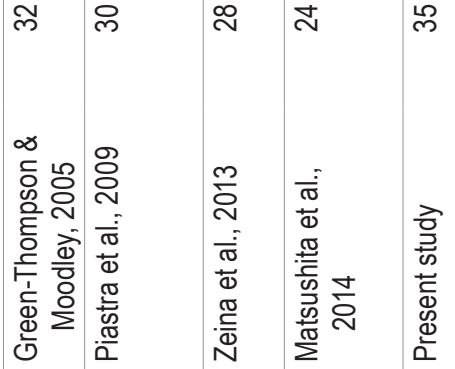


present case. The data are summarized in Table 1. The average gestational age at discovery of intracranial hemorrhage was 30.1 weeks (range 24-39 weeks). The mothers of 9 patients had an MVC as the mechanism of trauma, 3 mothers had been assaulted, and 2 mothers did not have a mechanism of trauma defined. Of the mothers involved in an MVC, 6 were wearing seat belts and 1 was not; information for 2 mothers was not available. Three mothers had air bags deploy and 1 did not; information for 5 mothers was not available. Five patients were delivered by emergent cesarean section, 2 by elective cesarean section, 2 by spontaneous vaginal delivery, 1 by induced vaginal delivery, and 1 birth was not specified. One patient was delivered using outlet forceps.

There were 12 patients with SDH, 1 with $\mathrm{EDH}$, and 1 with conflicting reports on location of hemorrhage (epidural or subdural). Two patients had predominantly posterior fossa SDH. One patient had an unusual supratentorial interhemispheric EDH. Three patients had associated skull fractures. Two patients had to undergo intervention for the intracranial hematoma.

In terms of the primary outcome of mortality, 3 patients had intrauterine fetal demise, and 3 died postpartum. The secondary outcome or neurological outcome showed that 3 patients had persistent neurological deficits ( 2 with hemiparesis and 1 with quadriparesis and cortical blindness), while 5 patients had no known neurological deficit at last follow-up.

\section{Discussion}

Blunt prenatal trauma resulting in fetal EDH or SDH is rarely reported in the literature. Ghi et al. previously investigated 109 cases of antenatally diagnosed intracranial hemorrhage, including 16 of their own cases. ${ }^{9}$ Of these cases, 89 were subependymal or intraventricular hemorrhages, while only 20 involved the subdural space. Antecedent maternal trauma was noted in 10 of the 109 cases (including 2 with SDH). A mortality rate of $40 \%$ was noted, including elective termination of pregnancy, intrauterine fetal demise, and death in the neonatal period. Of 48 patients with follow-up data, $52 \%$ were noted to be neurologically intact. Akman and Cracco investigated 32 reported cases of intrauterine SDH. ${ }^{1}$ They did not report any cases with clear antecedent trauma, although they did make note of 18 cases in which abdominal massage was performed prior to discovery of SDH..$^{11,12}$ Sherer et al. evaluated predisposing risk factors for antepartum fetal intracranial hemorrhage. ${ }^{23}$ They noted that severe maternal abdominal trauma, in addition to coagulopathies, seizures, cocaine abuse, amniocentesis, cholestasis of pregnancy, and febrile disease, accounted for known risk factors. The prognosis based on available literature for antenatally diagnosed intracranial hemorrhage has been poor. ${ }^{25}$

This systematic review of the literature revealed 13 previously published cases of fetal EDH or SDH that had clear antecedent maternal trauma. Interestingly, as was the case in our patient, most of the trauma described was mild. Most maternal injuries consisted of orthopedic fractures or soft-tissue bruising, and only 3 mothers (21\%) required operative intervention for trauma. It should be noted that there may be an inherent publication bias suggesting that fetal demise as a result of severe trauma is expected, and thus investigation into the incidence of intracranial hemorrhage in this population is not performed. The majority of patients in the present study had MVC as the method of trauma, but assault is a significant factor related to fetal EDH or SDH. The impact of seat belt use and air bag deployment on fetal health is poorly understood. ${ }^{16}$

As suggested by earlier reports, there was an overall poor prognosis with fetal EDH or SDH. There was a total mortality rate of $43 \%$, including intrauterine fetal demise and death in the neonatal period. It should be noted, however, that 7 children $(50 \%)$ had good neurological outcomes, with only 1 child noted to have a neurologically devastated outcome. This would suggest that discovery of fetal SDH or EDH may not lead to poor outcome by default. Green-Thompson and Moodley presented an interesting case in which literature review at that time caused the authors to offer a termination of pregnancy to a patient with fetal SDH due to poor prognosis. ${ }^{10}$ However, the patient went into spontaneous labor, and the infant had no neurological deficits noted at last follow-up.

Management of fetal SDH or EDH is varied among the cases presented here. Five mothers underwent emergent cesarean section, and 4 mothers had elective or planned deliveries. Of the elective deliveries, 2 were vaginal and 2 were via cesarean section. Currently, there is insufficient evidence to suggest the appropriate method of delivery. In the case of abnormal cardiotocography results, likely indicative of fetal distress after trauma, an urgent cesarean section is likely the best option. Maternal tachycardia and abnormal fetal heart rates are known risk factors for fetal demise after trauma. ${ }^{5}$ However, in the absence of these signs, a multidisciplinary approach with input from the mother to decide on timing and method of delivery is ideal. Only 2 patients had operative intervention for the intracranial hemorrhage. We suggest that these hemorrhages be managed by routine standard of care for other postnatal or neonatal SDH. In our presented case, we are electing to carefully monitor the patient with serial imaging, as the child has no known neurological deficits and does not have evidence of intracranial hypertension.

There are several limitations to this study. As mentioned previously, there is an inherent publication bias in the available literature. In addition, all of the included reports were retrospective case reports or small case series, with no higher level data available. Finally, there was inconsistent follow-up, potentially limiting the value of neurological outcome data. In the case presented here, the last follow-up was only at 3 months of age.

\section{Conclusions}

Fetal EDH or SDH is a rare outcome of maternal trauma with high mortality. However, good neurological outcomes are possible after the neonatal period.

\section{References}

1. Akman CI, Cracco J: Intrauterine subdural hemorrhage. Dev Med Child Neurol 42:843-846, 2000 
2. Baethmann M, Kahn T, Lenard HG, Voit T: Fetal CNS damage after exposure to maternal trauma during pregnancy. Acta Paediatr 85:1331-1338, 1996

3. Barozzino T, Sgro M, Toi A, Akouri H, Wilson S, Yeo E, et al: Fetal bilateral subdural haemorrhages. Prenatal diagnosis and spontaneous resolution by time of delivery. Prenat Diagn 18:496-503, 1998

4. Breysem L, Cossey V, Mussen E, Demaerel P, Van de Voorde W, Smet M: Fetal trauma: brain imaging in four neonates. Eur Radiol 14:1609-1614, 2004

5. Curet MJ, Schermer CR, Demarest GB, Bieneik EJ III, Curet LB: Predictors of outcome in trauma during pregnancy: identification of patients who can be monitored for less than 6 hours. J Trauma 49:18-25, 2000

6. El-Kady D, Gilbert WM, Anderson J, Danielsen B, Towner D, Smith LH: Trauma during pregnancy: an analysis of maternal and fetal outcomes in a large population. Am J Obstet Gynecol 190:1661-1668, 2004

7. Ellestad SC, Shelton S, James AH: Prenatal diagnosis of a trauma-related fetal epidural hematoma. Obstet Gynecol 104:1298-1300, 2004

8. Ford RM, Picker RH: Fetal head injury following motor vehicle accident; an unusual case of intrauterine death. Aust N Z J Obstet Gynaecol 29:72-73, 1989

9. Ghi T, Simonazzi G, Perolo A, Savelli L, Sandri F, Bernardi $\mathrm{B}$, et al: Outcome of antenatally diagnosed intracranial hemorrhage: case series and review of the literature. Ultrasound Obstet Gynecol 22:121-130, 2003

10. Green-Thompson R, Moodley J: In-utero intracranial haemorrhage probably secondary to domestic violence: case report and literature review. J Obstet Gynaecol 25:816-818, 2005

11. Gunn TR, Becroft DM: Unexplained intracranial haemorrhage in utero: the battered fetus? Aust $N$ Z J Obstet Gynaecol 24:17-22, 1984

12. Gunn TR, Mok PM, Becroft DM: Subdural hemorrhage in utero. Pediatrics 76:605-610, 1985

13. Hagmann CF, Schmitt-Mechelke T, Caduff JH, Berger TM: Fetal intracranial injuries in a preterm infant after maternal motor vehicle accident: a case report. Pediatr Crit Care Med 5:396-398, 2004

14. Hayes B, Ryan S, Stephenson JB, King MD: Cerebral palsy after maternal trauma in pregnancy. Dev Med Child Neurol 49:700-706, 2007

15. Karimi P, Ramus R, Urban J, Perlman JM: Extensive brain injury in a premature infant following a relatively minor maternal motor vehicle accident with airbag deployment. J Perinatol 24:454-457, 2004

16. Matsushita H, Harada A, Sato T, Kurabayashi T: Fetal intracranial injuries following motor vehicle accidents with airbag deployment. J Obstet Gynaecol Res 40:599-602, 2014

17. Matthews G, Hammersley B: A case of maternal pelvic trauma following a road traffic accident, associated with fetal intracranial haemorrhage. J Accid Emerg Med 14:115-117, 1997

18. Mirza FG, Devine PC, Gaddipati S: Trauma in pregnancy: a systematic approach. Am J Perinatol 27:579-586, 2010

19. Moher D, Shamseer L, Clarke M, Ghersi D, Liberati A, Petticrew M, et al: Preferred reporting items for systematic review and meta-analysis protocols (PRISMA-P) 2015 statement. Syst Rev 4:1, 2015

20. Murphy NJ, Quinlan JD: Trauma in pregnancy: assessment, management, and prevention. Am Fam Physician 90:717722,2014

21. Palmer JD, Sparrow OC: Extradural haematoma following intrauterine trauma. Injury 25:671-673, 1994

22. Piastra M, Pietrini D, Massimi L, Caldarelli M, De Luca D, Del Lungo LM, et al: Severe subdural hemorrhage due to minimal prenatal trauma. J Neurosurg Pediatr 4:543-546, 2009

23. Sherer DM, Anyaegbunam A, Onyeije C: Antepartum fetal intracranial hemorrhage, predisposing factors and prenatal sonography: a review. Am J Perinatol 15:431-441, 1998

24. Theurer DE, Kaiser IH: Traumatic fetal death without uterine injury. Report of a case. Obstet Gynecol 21:477-480, 1963

25. Vergani P, Strobelt N, Locatelli A, Paterlini G, Tagliabue P, Parravicini E, et al: Clinical significance of fetal intracranial hemorrhage. Am J Obstet Gynecol 175:536-543, 1996

26. Zeina AR, Kessel B, Mahamid A, Gazmawi J, Shrim A, Nachtigal A, et al: Computed tomographic diagnosis of traumatic fetal subdural hematoma. Emerg Radiol 20:169-172, 2013

\section{Disclosures}

The authors report no conflict of interest concerning the materials or methods used in this study or the findings specified in this paper.

\section{Author Contributions}

Conception and design: Joseph. Acquisition of data: Joseph. Analysis and interpretation of data: Joseph, Smith. Drafting the article: all authors. Critically revising the article: all authors. Reviewed submitted version of manuscript: Joseph, Garton. Approved the final version of the manuscript on behalf of all authors: Joseph. Statistical analysis: Joseph.

\section{Correspondence}

Jacob R. Joseph, Department of Neurosurgery, University of Michigan, 1500 East Medical Center Dr., Rm. 3553 TC, Ann Arbor, MI 48109-5338. email: jojacob@med.umich.edu. 